\title{
Lower bound plane stress element for modelling 3D structures
}

Herfelt, Morten Andersen; Poulsen, Peter Noe; Hoang, Linh Cao; Jensen, Jesper Frøbert

Published in:

Proceedings of the Institution of Civil Engineers - Engineering and Computational Mechanics

Link to article, DOI:

10.1680/jencm.16.00026

Publication date:

2017

Document Version

Peer reviewed version

Link back to DTU Orbit

Citation (APA):

Herfelt, M. A., Poulsen, P. N., Hoang, L. C., \& Jensen, J. F. (2017). Lower bound plane stress element for modelling 3D structures. Proceedings of the Institution of Civil Engineers - Engineering and Computational Mechanics, 170(3), 107-117. https://doi.org/10.1680/jencm.16.00026

\section{General rights}

Copyright and moral rights for the publications made accessible in the public portal are retained by the authors and/or other copyright owners and it is a condition of accessing publications that users recognise and abide by the legal requirements associated with these rights.

- Users may download and print one copy of any publication from the public portal for the purpose of private study or research.

- You may not further distribute the material or use it for any profit-making activity or commercial gain

- You may freely distribute the URL identifying the publication in the public portal

If you believe that this document breaches copyright please contact us providing details, and we will remove access to the work immediately and investigate your claim. 


\section{Lower bound plane stress element for modelling of 3D structures}

\section{A. Herfelt, MSc}

NIRAS A/S, Teknikerbyen 34, 2830 Virum, Denmark Department of Civil Engineering, Technical University of Denmark, Brovej, Building 118, 2800 Kgs. Lyngby, Denmark

\section{P. N. Poulsen, Phd}

Department of Civil Engineering, Technical University of Denmark, Brovej, Building 118, 2800 Kgs. Lyngby, Denmark

\author{
L. C. Hoang, Phd \\ Department of Civil Engineering, Technical University of Denmark, \\ Brovej, Building 118, 2800 Kgs. Lyngby, Denmark
}

J. F. Jensen, lic.techn.

NIRAS A/S, Teknikerbyen 34, 2830 Virum, Denmark

In-plane action is often the primary load carrying mechanism of reinforced concrete structures. The plate bending action will be secondary, and the behaviour of the structure can be modelled with a reasonable accuracy using a generalised plane stress element for three-dimensions. In this paper, the formulation of such element is given and the Mohr-Coulomb and von Mises criteria are presented for second-order cone programming. Three examples of increasing complexity are used to analyse the performance of the element and the convergence rate and demonstrate the potential of the proposed element.

\section{Introduction}

The lateral stability of reinforced concrete structures is often ensured by shear walls. The horizontal loads, e.g. wind or seismic loads, are transferred as in-plane forces via the concrete slabs and the shear walls to the foundations of the structure. The in-plane forces are transferred as shear between the slabs and shear walls as well as in-between shear walls. The transverse forces acting on the slabs and facades are in this regard secondary to the in-plane forces. It is crucial to the overall capacity of the structure that the analysis considers the structural system as a single unit: If the shear walls are analysed individually, a significant portion of the strength is neglected, see Fig. 1.

Practical design and analysis of reinforced concrete structures in the ultimate limit state often requires consideration of plastic material behaviour. This material behaviour can be incorporated in the analysis by use of either simplified models, e.g. rigid-plasticity (see e.g. Drucker et al., 1952; Prager, 1952), or more advanced model which may include hardening, softening, etc. The latter can be implemented in numerical frameworks, e.g. finite element
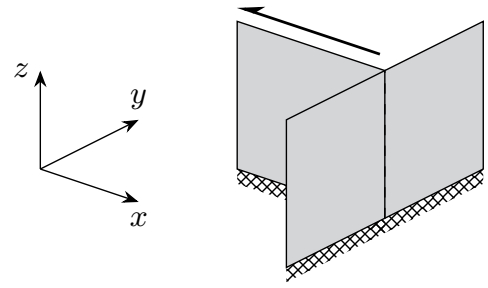

Figure 1. Shear wall subjected to a shear force: The transverse wall increases the capacity of the structure.

analysis, and provides the most accurate results compared to the simplified material models. While the advanced models can model the observed material behaviour to a reasonable degree, it is often difficult to obtain the required material parameters for the models.

Models based on the theory of rigid-plasticity have been used for almost a century to assess the capacity of concrete structures (Ingerslev, 1921; Nielsen and Hoang, 2010). The rather crude material model leads to an elegant framework known as limit analysis in which several methods have been developed e.g. homogeneous stress triangles (Nielsen, 1971), the yield line method (Johansen, 1962), and stress field methods (Muttoni et al., 1997). 
The methods can be classified as either upper bound methods, where a kinematically admissible displacement field is determined, or as lower bound methods, where a statically admissible stress field is determined. In practice, manual upper bound and lower bound methods are still widely used to assess the capacity in the ultimate limit state. The accuracy of the calculations, however, is very dependent on the skill and intuition of the individual structural engineer. For complex structures, the results may be far from the actual capacity.

Finite element limit analysis is the numerical counterpart of manual limit analysis. The method is a special case of the general finite element method and assumes a rigid-plastic material behaviour. Like manual limit analysis, finite element limit analysis can be formulated either as lower bound, upper bound problems, or mixed problems (which are often more accurate than the strict upper and lower bound problems). Anderheggen and Knöpfel (1972) presented the general framework as well as finite elements for both solids and plate bending. The mathematical problem of finite element limit analysis is formulated as a convex optimisation problem which can be solved remarkably efficiently using state-ofthe-art solvers.

Several authors have treated plane stress and plane strain elements (see e.g. Sloan, 1988; Poulsen and Damkilde, 2000; Makrodimopoulos and Martin, 2006, 2007). Plane strain elements, however, have received most of the attention as they are used in geotechnical engineering. More recently, meshless methods have been presented as an alternative to the classical finite element version of limit analysis (Smith et al., 2014). Adaptive meshing has a major potential for finite element limit analysis as displayed by Lyamin et al. (2005) amongst others. Numerical limit analysis of concrete structures have not received the same amount of attention, but there have nevertheless been some attempts at treating threedimensional concrete structures within the framework of finite element limit analysis (Larsen, 2010).

This paper will present the basic mathematical formulation of finite element limit analysis, namely lower bound load optimisation. A brief introduction to second-order cone programming (SOCP) is given and solution strategies will be presented.

The basic lower bound plane stress element is generalised to three-dimensions, which will make it possible to model the load carrying systems of modern concrete buildings in a simple manner while disregarding the plate bending behaviour. For reinforced concrete, the proposed element will use the Mohr-Coulomb yield criterion, which can be cast as second-order cones, hence, the final optimisation problem will be a second-order cone program. Three examples will be presented: The first example will be used to validate the implementation and analyse the convergence rate of the element, while the second and third examples will demonstrate the use and strength of the proposed element.

\section{Convex optimisation and limit analysis}

\subsection{Convex optimisation}

Convex optimisation problems, also known as convex programs, can be found within several engineering applications, e.g. antenna ray weight design and truss optimisation (Lobo et al., 1998). The main advantage of convex optimisation is that any optimum will be the global optimum, hence, the class of problems can be solved efficiently using gradient based methods. For general non-linear optimisation problems, several local extrema may exist making it practically impossible to ensure that the found solution is the global extremum. For plane problems in finite element limit analysis, second-order cone programming is often used as the commonly used yield functions can be represented exact using second-order constraints (Bisbos and Pardalos, 2007).

Second-order cone programs are non-linear convex optimisation problems, where a linear objective function is minimised over the intersection of an affine set and the Cartesian product of secondorder cones (Andersen et al., 2003). The standard form of SOCP can be stated as:

$$
\begin{array}{ll}
\text { minimise } & \boldsymbol{g}^{T} \boldsymbol{x} \\
\text { subject to } & \mathbf{A} \boldsymbol{x}=\boldsymbol{b}, \\
& \boldsymbol{x} \in \mathcal{Q}
\end{array}
$$

where $\boldsymbol{x}$ is the problem variables. The matrix, $\mathbf{A}$, and two vectors, $\boldsymbol{b}$ and $\boldsymbol{g}$, define the linear constraints and objective function of the optimisation problem. The notation, $\boldsymbol{x} \in \mathcal{Q}$, indicates that the vector $\boldsymbol{x}$ should be in the Cartesian product of second-order cones, i.e.:

$$
\boldsymbol{x} \in \mathcal{Q} \quad \Leftrightarrow \quad \boldsymbol{x}_{1} \in \mathcal{Q}_{m_{1}}, \boldsymbol{x} x_{2} \in \mathcal{Q}_{m_{2}}, \ldots, \boldsymbol{x}_{q} \in \mathcal{Q}_{m_{q}}
$$

where $\boldsymbol{x}_{i}$ are subvectors of $\boldsymbol{x}$ and $\mathcal{Q}_{m_{i}}$ is a quadratic cone of size $m_{i}$. All second-order cone programs can be recast to fit the standard form (1) by e.g. adding slack variables. The simplest quadratic cone is the second-order cone also known as the Lorentz cone, which can 
be stated as the following set:

$$
\mathcal{Q}:=\left\{\boldsymbol{x} \in \mathbb{R}^{n}: x_{1}^{2} \geq \sum_{i=2}^{n} x_{i}^{2}, x_{1} \geq 0\right\}
$$

All quadratic cones can be transformed to the second-order cone (3).

SOCP can be solved efficiently by interior point methods, a class of algorithms developed from the polynomial time algorithm proposed by Karmarkar (1984). Interior point methods are based on a steepest descend approach, and in state-of-the-art solvers the Karush-KuhnTucker (KKT) conditions of the original optimisation problem is embedded in a slightly larger model, a so-called homogeneous model (see e.g. Nesterov et al., 1999; Sturm, 1997), which makes it possible to easily detect primal and dual infeasibility as well as illposed problems. The homogeneous model is solved using Newton's method, however, the step size is restricted to very small steps. Nesterov-Todd scaling (see Nesterov and Todd, 1997) is used to facilitate longer steps, and the search direction is computed in a scale space where it is uniquely defined.

Modern solvers are capable of solving large scale optimisation problems with hundreds of thousands variables and constraints in a matter of minutes on a standard laptop due to the polynomial time complexity of the algorithm. Large scale finite element limit analysis problems will be extremely sparse, which can be exploited by solvers to reduce the time complexity to near linear. This a major advantage over non-linear finite element analysis, which often requires much longer computational times. For an in-depth description of convex optimisation and state-of-the-art solvers, the reader is referred to Boyd and Vandenberghe (2004), Andersen et al. (2003), and Terlaky (2013).

\subsection{Lower bound limit analysis}

The scope of lower bound limit analysis is to maximise the variable load acting on the structure while ensuring a statically admissible stress field, i.e. a stress field which satisfy equilibrium and does not violate the yield criterion in any point. The objective function of the optimisation problem is the load factor, $\lambda$, which is sought to be maximised.

Every optimisation problem has a so-called dual problem, which is linked to the original (primal) problem via the Lagrange function and KKT conditions. The dual problem of lower bound limit analysis is the corresponding kinematic problem. The primal and dual problems are solved simultaneously, and while the solution to the lower bound problem yields a statically admissible stress field, the solution to the corresponding kinematic problem can be interpreted as the collapse mode.

The mathematical problem of lower bound load optimisation can be derived from the virtual work equation and can be stated as follows:

$$
\begin{array}{ll}
\text { maximise } & \lambda \\
\text { subject to } & \mathbf{B}^{T} \boldsymbol{\sigma}=\boldsymbol{p} \lambda+\boldsymbol{p}_{0} \\
& f\left(\boldsymbol{\sigma}_{i}\right) \leq 0, i=1,2, \ldots, m
\end{array}
$$

The linear equilibrium equations and yield criteria ensure a statically admissible stress field while the load factor $\lambda$ is maximised. The structure is subject to a load composed by a fixed part, $\boldsymbol{p}_{0}$, and a scalable part, $\boldsymbol{p} \lambda . \mathbf{B}^{T}$ is the global equilibrium matrix and $\sigma$ is the stress vector. The yield function $f$ is generally non-linear, but convex, hence, the problem (4) is a convex optimisation problem. For plane problems, the MohrCoulomb criterion with a tension cut-off can be cast as second-order cones. Second-order cone programming have been used for more than a decade in the field of finite element limit analysis (Bisbos and Pardalos, 2007; Krabbenhøft et al., 2007; Makrodimopoulos and Martin, 2007) and can be considered as an established technology at this point.

Assuming that the yield function $f$ can be represented by linear and second-order constraints, the problem (4) can be expanded to obtain the following form:

$$
\begin{array}{ll}
\operatorname{maximise} & \lambda \\
\text { subject to } & \mathbf{B}^{T} \boldsymbol{\sigma}=\boldsymbol{p} \lambda+\boldsymbol{p}_{0} \\
& \mathbf{C}_{\beta} \boldsymbol{\beta}+\mathbf{C}_{\alpha} \boldsymbol{\alpha}+\mathbf{C}_{\gamma} \boldsymbol{\gamma}=\boldsymbol{C}_{0} \\
& \mathbf{E}_{\sigma} \boldsymbol{\beta}+\mathbf{E}_{\alpha} \boldsymbol{\alpha}+\mathbf{E}_{\gamma} \boldsymbol{\gamma} \leq \boldsymbol{E}_{0} \\
& \boldsymbol{\gamma}_{i} \in \mathcal{Q}_{k_{i}}, \quad i=1,2, \ldots, m,
\end{array}
$$

where $\mathbf{C}_{\sigma}, \mathbf{C}_{\alpha}$, and $\mathbf{C}_{\gamma}$ are matrices associated with the linear equality constraints for the yield function, while the matrices, $\mathbf{E}_{\sigma}$, $\mathbf{E}_{\alpha}$, and $\mathbf{E}_{\gamma}$, define the linear inequality constraints. The two vectors $\boldsymbol{C}_{0}$ and $\boldsymbol{E}_{0}$ typically contain material parameters. The variable vectors, $\boldsymbol{\alpha}$ and $\gamma$, contain the auxiliary variables used for the yield function, whereas $\gamma$ is used for the second-order constrains: The vector $\gamma_{i}$ is a subset of $\gamma$ associated with the $i$ th checkpoint which is required to be in a quadratic cone $\mathcal{Q}_{k_{i}}$ of size $k_{i}$. The scalar $m$ is the number of checkpoints. 


\section{Finite element formulation}

\subsection{Lower bound plane stress element}

The geometry of the element is defined by three corner nodes. A linear stress field is chosen for the element, and a set of stress variables which describe a plane stress state is associated with each of the three nodes. The stresses of the element are given in the local coordinate system of the element. The element stress vector is given as

(6) $\boldsymbol{\sigma}_{e l}=\left[\begin{array}{l}\boldsymbol{\sigma}_{1} \\ \boldsymbol{\sigma}_{2} \\ \boldsymbol{\sigma}_{3}\end{array}\right]$,

where $\sigma_{i}$ is the set of stress variables associated with the $i$ th node,

$$
\boldsymbol{\sigma}_{i}=\left[\begin{array}{c}
\sigma_{x}^{i} \\
\sigma_{y}^{i} \\
\tau_{x y}^{i}
\end{array}\right]
$$

The element requires a total of nine variables to describe the linear stress field.

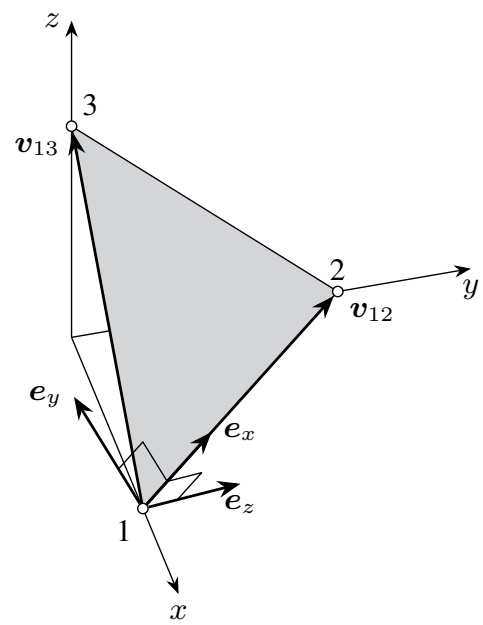

Figure 2. Geometry and local coordinate system of the three-dimensional element.

The local coordinate system of the given element is defined by the following basis vectors:

(8) $\quad \boldsymbol{e}_{z}=\frac{\boldsymbol{v}_{12} \times \boldsymbol{v}_{13}}{\left\|\boldsymbol{v}_{12} \times \boldsymbol{v}_{13}\right\|}, \quad \boldsymbol{e}_{x}=\frac{\boldsymbol{v}_{12}}{\left\|\boldsymbol{v}_{12}\right\|}, \quad \boldsymbol{e}_{y}=\boldsymbol{e}_{z} \times \boldsymbol{e}_{x}$,

where $\boldsymbol{v}_{i j}$ is a vector going from node $i$ to node $j$ in the global coordinates. The local coordinates of node $i, \boldsymbol{x}_{i}$, can now be determined by the transformation,

(9) $\quad \boldsymbol{x}_{i}=\mathbf{E}^{T} \boldsymbol{X}_{i}, \quad$ with $\quad \mathbf{E}=\left[\begin{array}{lll}\boldsymbol{e}_{x} & \boldsymbol{e}_{y} & \boldsymbol{e}_{z}\end{array}\right]$

where $\boldsymbol{X}_{i}$ is the global coordinates of node $i$, and $\mathbf{E}$ is the transformation matrix. For each element boundary, we define a normal vector in the local coordinate system,

(10) $\boldsymbol{n}_{i}=\left[\begin{array}{l}n_{x}^{i} \\ n_{y}^{i}\end{array}\right]$,

where $i$ is the element side number. In order to obtain a lower bound solution, traction continuity is required. Due to the linear stress field, equilibrium of tractions have to be enforced twice for each element side. Based on the normal vectors (10), we define the stress-to-traction array $\mathbf{P}_{i}^{T}$ for side $i$,

(11) $\mathbf{P}_{i}^{T}=\left[\begin{array}{ccc}n_{x}^{i} & 0 & -n_{y}^{i} \\ 0 & -n_{y}^{i} & n_{x}^{i} \\ 0 & 0 & 0\end{array}\right]$

and

(12) $\tilde{\mathbf{P}}_{i}^{T}=l_{i} \mathbf{P}_{i}^{T}$,

where $l_{i}$ is the length of side $i$. The last row of $\mathbf{P}_{i}^{T}$ represent the local $z$-direction, in which no tractions are present. The global equilibrium of the system is done in global coordinates, hence, it is necessary to transform the tractions. The tractions in global coordinates for node $i$ of the element can therefore be stated as

(13) $\boldsymbol{q}_{i}=\left[\begin{array}{l}q_{j x}^{i} \\ q_{j y}^{i} \\ q_{j z}^{i} \\ q_{k x}^{i} \\ q_{k y}^{i} \\ q_{k z}^{i}\end{array}\right]=\frac{1}{2}\left[\begin{array}{ll}\mathbf{E}^{T} & \\ & \mathbf{E}^{T}\end{array}\right]\left[\begin{array}{c}\tilde{\mathbf{P}}_{j}^{T} \\ \tilde{\mathbf{P}}_{k}^{T}\end{array}\right] \boldsymbol{\sigma}_{i}$

where $j$ and $k$ are the two sides which meet in node $i$, and $q_{j x}^{i}$ is the traction in the $x$-direction on side $j$ at node $i$ of the element.

The element can be subjected to surface loads, $\gamma_{x}$ and $\gamma_{y}$ in the local $x$ and $y$-directions, acting on the entire area. The derivatives of the linear stress field must balance these surface loads, which 
leads to two additional equilibrium equations:

$$
\begin{aligned}
& \frac{\partial \sigma_{x}}{\partial x}+\frac{\partial \tau_{x y}}{\partial y}+\gamma_{x}=0 \\
& \frac{\partial \sigma_{y}}{\partial y}+\frac{\partial \tau_{x y}}{\partial x}+\gamma_{y}=0
\end{aligned}
$$

Utilising the shape functions of the linear stress field, the so-called internal equilibrium equations (14) can now be stated as

$$
\boldsymbol{q}_{c}=A\left[\begin{array}{c}
\gamma_{x} \\
\gamma_{y}
\end{array}\right]=\frac{1}{2}\left[\begin{array}{lll}
\tilde{\mathbf{P}}_{1}^{T} & \tilde{\mathbf{P}}_{2}^{T} & \tilde{\mathbf{P}}_{3}^{T}
\end{array}\right] \sigma
$$

The element equilibrium matrix can be written as follows by combining (13) and (15):

(16) $\boldsymbol{q}=\frac{1}{2}\left[\begin{array}{ccc}\mathbf{E}^{T} \tilde{\mathbf{P}}_{2}^{T} & & \\ \mathbf{E}^{T} \tilde{\mathbf{P}}_{3}^{T} & & \\ & \mathbf{E}^{T} \tilde{\mathbf{P}}_{3}^{T} & \\ & \mathbf{E}^{T} \tilde{\mathbf{P}}_{1}^{T} & \\ & & \mathbf{E}^{T} \tilde{\mathbf{P}}_{1}^{T} \\ & & \mathbf{E}^{T} \tilde{\mathbf{P}}_{2}^{T} \\ \tilde{\mathbf{P}}_{1}^{T} & \tilde{\mathbf{P}}_{2}^{T} & \tilde{\mathbf{P}}_{3}^{T}\end{array}\right]\left[\begin{array}{c}\boldsymbol{\sigma}_{1} \\ \boldsymbol{\sigma}_{2} \\ \boldsymbol{\sigma}_{3}\end{array}\right]=\mathbf{B}_{e l}^{T} \boldsymbol{\sigma}_{e}$

Lower bound elements may contain linear dependencies which cause numerical issues (Makrodimopoulos and Martin, 2006) These problems, however, can be avoided by dividing the element into three subelements, each with a linear stress field. This was done by Herfelt et al. (2016) for the lower bound plane stress element by Poulsen and Damkilde (2000). The subdivision also increase the accuracy of the element, however, the problem size is likewise increased. Several of the additional variables and equations can be eliminated, hence, the problem size is only increased marginally.

\subsection{Reinforced concrete yield criterion}

The yield criterion is enforced for all three sets of stresses of the element to ensure a safe stress field. For the equilibrium equations the total stresses are used, which comprise the stresses carried by the concrete and by the reinforcement. The Mohr-Coulomb yield criterion with a tension cut-off is used for the concrete, while a simple, linear criterion is adopted for the reinforcement.

The reinforcement is assumed to consist of an orthogonal mesh of rebars oriented in an angle $\theta$ to the local coordinate system of the element, see Fig. 3. Moreover, it is assumed that the reinforcement only carries axial forces (Nielsen and Hoang, 2010). The relation between the total stresses, the concrete stresses, and

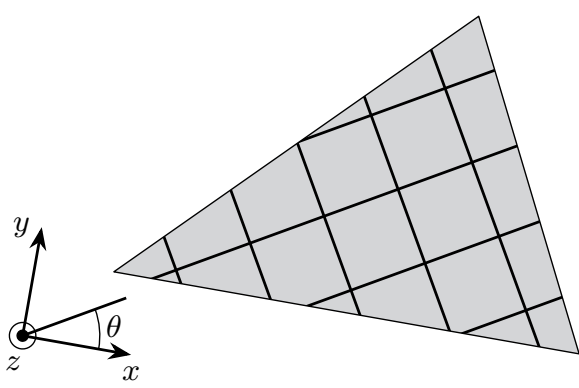

Figure 3. Orthogonal reinforcement in an angle $\theta$ to the local coordinate system of the element.

the reinforcement stresses are given as

$$
\left[\begin{array}{ccc}
c^{2} & s^{2} & 2 s c \\
s^{2} & c^{2} & -2 s c \\
-s c & s c & c^{2}-s^{2}
\end{array}\right]\left[\begin{array}{c}
\sigma_{x} \\
\sigma_{y} \\
\tau_{x y}
\end{array}\right]=\left[\begin{array}{c}
\sigma_{x m} \\
\sigma_{y m} \\
\tau_{x y m}
\end{array}\right]+\left[\begin{array}{c}
\tilde{\sigma}_{x s} \\
\tilde{\sigma}_{y s} \\
0
\end{array}\right],
$$

where $c=\cos \theta$ and $s=\sin \theta$. Subscript $m$ indicates concrete stresses while subscript $s$ indicates reinforcement stresses. Moreover, $\tilde{\sigma}_{s i}$ is the equivalent reinforcement stress defined as

(18) $\tilde{\sigma}_{s i}=\frac{A_{s i}}{t} \sigma_{s i}$

where $A_{s i}$ is the reinforcement area per unit length in the $i$-direction and $t$ is the out-of-plane thickness of the considered element. The yield criterion for the reinforcement can be written as follows using equivalent stresses:

$$
\begin{aligned}
& 0 \leq \tilde{\sigma}_{x s} \leq \tilde{f}_{y x}=\frac{A_{s x}}{t} f_{y} \\
& 0 \leq \tilde{\sigma}_{y s} \leq \tilde{f}_{y y}=\frac{A_{s y}}{t} f_{y}
\end{aligned}
$$

where $\tilde{f}_{y}$ is the equivalent yield strength. The compressive strength of the reinforcement is neglected as seen in (19), and the reinforcement stresses must be non-negative as a consequence.

The Mohr-Coulomb criterion is given in terms of principal stresses and can be stated as follows for plane stress:

$$
\sigma_{1} \leq f_{t}
$$

$$
\begin{aligned}
k \sigma_{1}-\sigma_{2} & \leq f_{c} \\
-\sigma_{2} & \leq f_{c}
\end{aligned}
$$

where $f_{t}$ is the uniaxial tensile strength of the concrete, and $f_{c}$ is the uniaxial compressive strength. $k$ is a friction parameter, which is usually taken as 4 for normal strength concrete corresponding to 
an angle of internal friction of approximately $37^{\circ} . \sigma_{1}$ and $\sigma_{2}$ are the largest and smallest principal stresses, respectively, which is given as

$$
\left.\begin{array}{l}
\sigma_{1} \\
\sigma_{2}
\end{array}\right\}=\frac{\sigma_{x m}+\sigma_{y m}}{2} \pm \sqrt{\left(\frac{\sigma_{x m}-\sigma_{y m}}{2}\right)^{2}+\tau_{x y m}^{2}}
$$

Introducing three auxiliary variables,

$$
\text { (22) } \begin{aligned}
\sigma_{d} & =\frac{\sigma_{x m}-\sigma_{y m}}{2}, \\
\varphi & \geq \sqrt{\sigma_{d}^{2}+\tau_{x y m}^{2}}
\end{aligned}
$$

bounds to the principal stresses (21) can now be stated:

$$
\begin{aligned}
\sigma_{1} & \leq-p_{m}+\varphi \\
-\sigma_{2} & \leq p_{m}+\varphi
\end{aligned}
$$

The yield criterion (20) can be written as three linear inequality constraints in addition to the definitions of the three auxiliary variables (22):

$$
\begin{aligned}
-p_{m}+\varphi & \leq f_{t} \\
(1-k) p_{m}+(k+1) \varphi & \leq f_{c} \\
p_{m}+\varphi & \leq f_{c}
\end{aligned}
$$

The yield criterion fits the form of second-order cone programming since the definition of $\varphi$ is a second-order cone (3).

\section{3. von Mises yield criterion}

The von Mises yield criterion is commonly used for metals and is based on the second stress invariant, $J_{2}$, which is given as follows:

$$
\begin{aligned}
J_{2}=\frac{\left(\sigma_{x}-\sigma_{y}\right)^{2}}{6} & +\frac{\left(\sigma_{y}-\sigma_{z}\right)^{2}}{6}+\frac{\left(\sigma_{z}-\sigma_{x}\right)^{2}}{6} \\
& +\tau_{x y}^{2}+\tau_{y z}^{2}+\tau_{x z}^{2}
\end{aligned}
$$

For plane stress, the second stress invariant is reduced to

(26) $J_{2}=\frac{\left(\sigma_{x}-\sigma_{y}\right)^{2}}{6}+\frac{\sigma_{y}^{2}}{6}+\frac{\sigma_{x}^{2}}{6}+\tau_{x y}^{2}$

The von Mises criterion is given as

(27) $\sqrt{3 J_{2}} \leq f_{y}$, where $f_{y}$ is the uniaxial yield strength. Introducing three auxiliary variables,

(28) $\alpha_{1}=\frac{\sqrt{3}}{2}\left(\sigma_{x}-\sigma_{y}\right), \alpha_{2}=\frac{1}{2}\left(\sigma_{x}+\sigma_{y}\right), \alpha_{3}=\sqrt{3} \tau_{x y}$, the criterion (27) can be restated as a second-order cone:

(29) $\sqrt{\alpha_{1}^{2}+\alpha_{2}^{2}+\alpha_{3}^{2}} \leq f_{y}$

It has been shown that both the reinforced concrete yield criterion and the von Mises yield criterion fit the format of second-order cone programming for plane stress.

\section{Examples}

The scope of this section is to analyse the performance of the element and illustrate the use. The commercial solver, MOSEK (MOSEK ApS, 2015) is used for the optimisation. For the computational time, please note that all calculations are performed on a laptop with an Intel core $i 7-4720 \mathrm{HQ}$ with $8 \mathrm{CPUs}$ and $2.6 \mathrm{GHz}$ clock frequency. The meshes for examples 2 and 3 are generated using GiD v12 (Ribó et al., 1998).

\subsection{Deep beam with shear supports}

The first example is a deep reinforced concrete beam subject to a uniformly distributed load. The beam is supported in either end by shear supports as seen in Fig 4. The analytical solution to the deep

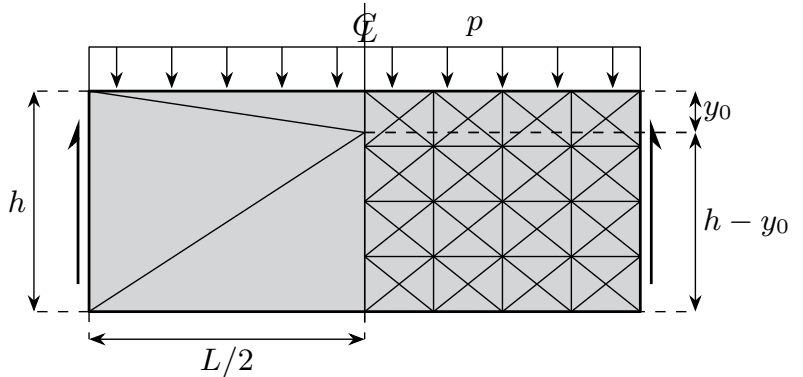

Figure 4. Deep beam with shear supports.

beam example is well-known (Nielsen and Hoang, 2010) and can be obtained by the mesh on the left side in Fig. 4:

(30) $\quad p^{*}=\frac{4 \Phi h^{2} f_{c}}{(1+\Phi) L^{2}}$

where $\Phi$ is the mechanical reinforcement ratio defined as:

(31) $\Phi=\frac{A_{s} f_{y}}{t f_{c}}$ 
Using $h=2 \mathrm{~m}, L=6 \mathrm{~m}, f_{c}=20 \mathrm{MPa}$, and $\Phi=0.075$, the exact limit load is $p^{*}=0.6202 \mathrm{MPa}$. A structured mesh (see the right hand side of Fig. 4) is used to calculate a lower bound of the limit load of the deep beam.

\begin{tabular}{cccc}
\hline$n e l$ & $p[\mathrm{MPa}]$ & Error $[\%]$ & Time $[\mathrm{s}]$ \\
\hline 64 & 0.5556 & 10.42 & 0.31 \\
256 & 0.6053 & 2.40 & 0.88 \\
1024 & 0.6177 & 0.39 & 3.77 \\
4096 & 0.6191 & 0.17 & 9.23 \\
16384 & 0.6193 & 0.13 & 43.40 \\
\hline
\end{tabular}

Table 1. Limit load, error, and computational time for the deep beam example.

Tab. 1 shows that the structured mesh approaches the exact limit load from below as the number of elements (nel) increases. The convergence and computational time will be discussed in a following section, but it is observed that the computational time appears to be roughly proportional to the problem size. The stress distribution is illustrated in Fig. 5.

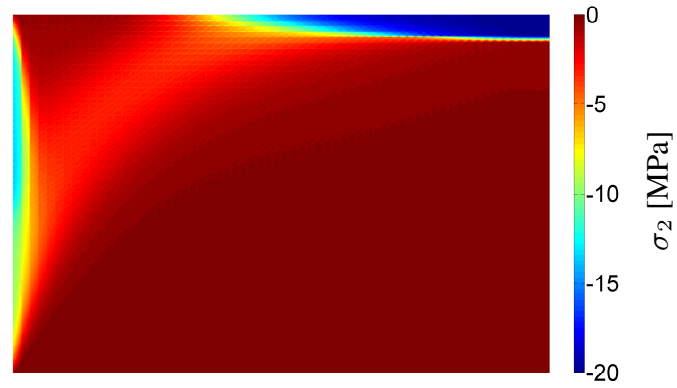

Figure 5. Lowest principal stress $\sigma_{2}$ for the deep beam example using 16384 elements.

\subsection{Cantilever I-beam}

A cantilever steel I-beam is subjected to a uniformly distributed line load acting on top of the web. The web has a height of 300 $\mathrm{mm}$, and the flanges have a width of $300 \mathrm{~mm}$. The web and flanges have a thickness of $10 \mathrm{~mm}$, and the cantilever beam has a length of 3 metres. The steel has a yield strength of $f_{y}=250 \mathrm{MPa}$. This gives a plastic moment capacity of $281 \mathrm{kNm}$ and a limit load of $p^{*}=62.5$ $\mathrm{kN} / \mathrm{m}$ assuming a maximum stress of $250 \mathrm{MPa}$. Four different meshes are analysed. The medium density mesh comprising 948 elements is shown in Fig. 6. With a thickness of just $10 \mathrm{~mm}$, the effect of local bending in the web and flanges is negligible, while

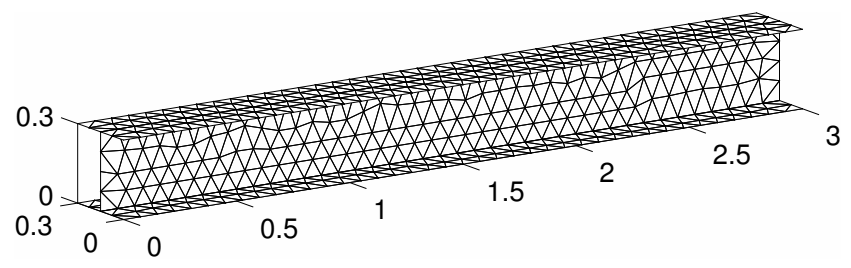

Figure 6. Medium density mesh of the cantilever I-beam using 948 elements. The beam is supported at the left end.

the external load will almost exclusively be carried via in-plane forces, hence, the proposed element will provide a decent estimate of the capacity.

The load capacity of the cantilever I-beam increases with the mesh

\begin{tabular}{lccc}
\hline Mesh & $n e l$ & $p[\mathrm{kN} / \mathrm{m}]$ & Time $[\mathrm{s}]$ \\
\hline Coarse & 238 & 63.24 & 0.44 \\
Medium & 948 & 64.18 & 1.86 \\
Fine & 3616 & 64.90 & 3.56 \\
Very fine & 14646 & 65.25 & 16.06 \\
\hline
\end{tabular}

Table 2. Limit load and computational time for the cantilever I-beam example.

density as seen in Tab. 2. The von Mises yield criterion requires fewer variables than the reinforced concrete criterion, hence, the computational time is lower for the same number of elements. The model predicts a limit load slightly larger than the analytical moment capacity, however, this is due to the von Mises criterion where the largest stress can exceed $f_{y}$ for certain stress states. Fig. 7 shows the largest and smallest principal stresses near the supported (left) end of the cantilever.

\subsection{Four-storey stairwell with door openings}

The third example is a four-storey stairwell of reinforced concrete with door openings. The stairwell is subjected to a shear force acting on top of the wall with the door openings (see Fig. 8), which causes both bending and torsion in the stairwell. The shear walls have a thickness of $180 \mathrm{~mm}$, hence, the effect of local bending in each individual wall is not negligible as in the previous example, however, a lower bound value is obtained by neglecting the moment capacity of the walls. In practice, the corners are reinforced with loop reinforcement which ensures the transfer of bending moments between adjacent walls. The dimensions of the shear walls are given in Fig. 8 and the door openings have a height of 2.10 metres and a 


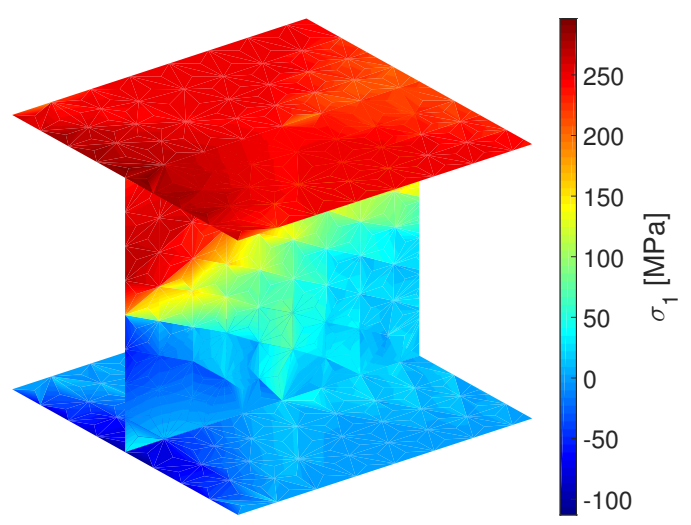

(a) $\sigma_{1}$

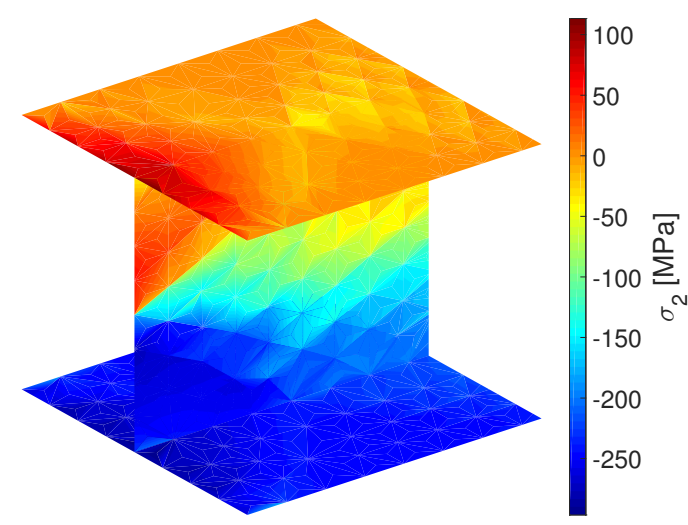

(b) $\sigma_{2}$

Figure 7. Largest and smallest principal stresses near the support of the I-beam using the fine mesh.

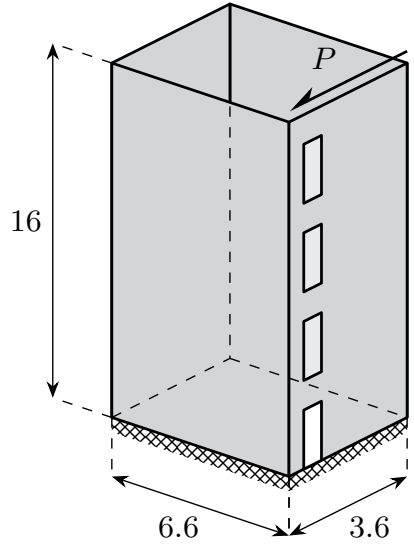

Figure 8. Four-storey shear wall subject to bending and torsion. Dimensions are given metres.

width of 0.90 metres. The shear walls are reinforced with two layers of $\varnothing 8$ bars per $150 \mathrm{~mm}$ in both directions. The design yield strength of the reinforcement is chosen as $f_{y d}=458 \mathrm{MPa}$. The concrete has a design compressive strength of $f_{c d}=21.43 \mathrm{MPa}$, while the tensile strength is set to zero. Two different effectiveness factors $\nu$ is considered, namely $\nu=1$ and $\nu=0.7-f_{c} / 200=0.550$ (where $f_{c}=30 \mathrm{MPa}$ is the characteristic strength), and the design compressive stress is reduced accordingly, $f_{c d}=\nu \cdot 21.43 \mathrm{MPa}$.

Tab. 3 shows that the coarse mesh yields a reasonable estimate despite using only 864 elements. The fine mesh yields less than 5 $\%$ additional capacity despite having 13 times more elements. It is noted that the model approaches the exact limit load from below which is to be expected from a lower bound element. Tab. 3 also

\begin{tabular}{lcccc}
\hline & \multicolumn{4}{c}{$p[\mathrm{kN} / \mathrm{m}]$} \\
Mesh & $n e l$ & $\nu=1$ & $\nu=0.550$ & Time [s] \\
\hline Coarse & 864 & 85.27 & 85.06 & 2.89 \\
Medium & 3564 & 88.62 & 87.80 & 10.52 \\
Fine & 11379 & 89.25 & 88.46 & 37.38 \\
\hline
\end{tabular}

Table 3. Limit load and computational time for the stairwell example.

shows that the two effectiveness factors yields approximately the same capacity: Using $\nu=0.550$ reduces the capacity by less than one percent since the reinforcement is the limiting factor.

Fig. 9(a) shows the collapse mode for the shear wall with door openings: The bending failure occurs near the supports, which allows the wall to start rotating. Moreover, local failures are observed near the door openings and the top. Fig. 9(b) to (e) shows the stress distribution for the four walls of the stairwell. It is seen that all walls mobilised and carry stresses. Struts are formed between the door openings as shown in Figure 9(c), and the slender columns to the left of the door openings carry considerably stresses. The largest compressive stresses occur near the bottom door opening and approach the compressive strength of the concrete, see Figure 9(b) and (c).

\subsection{Computational time and convergence}

The three examples have demonstrated the strength of the element. For all three examples, the capacity increased with the number of elements, i.e. the models approached the exact limit load from 


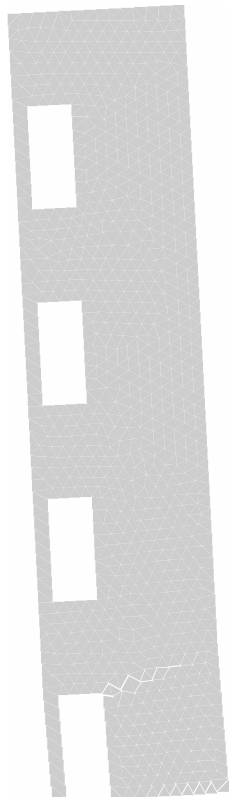

(a)

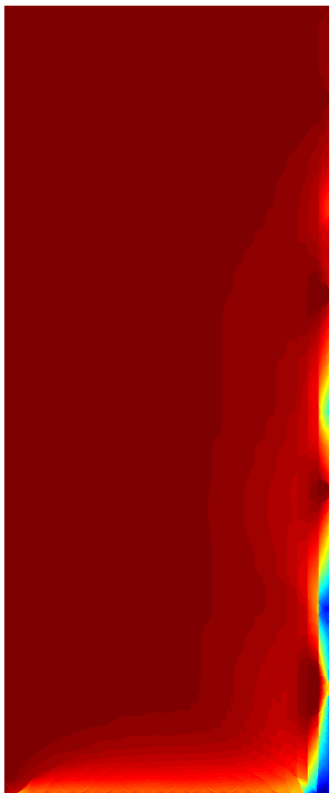

(b)

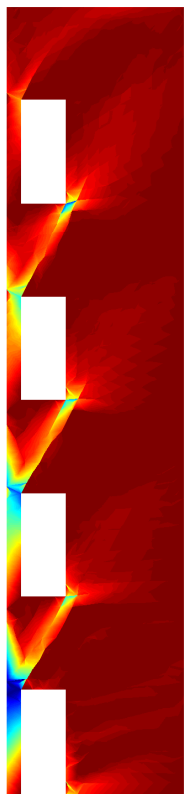

(c)

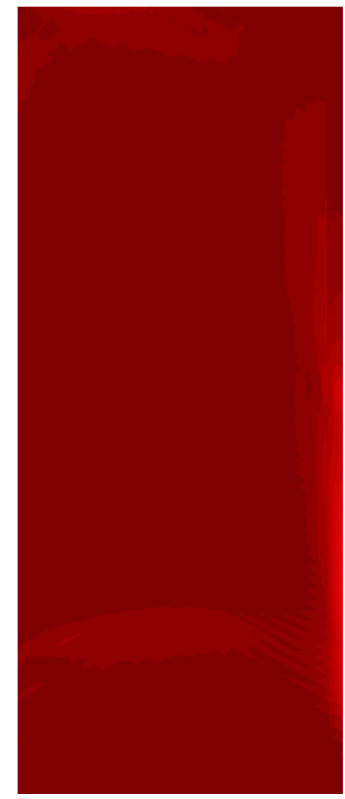

(d)

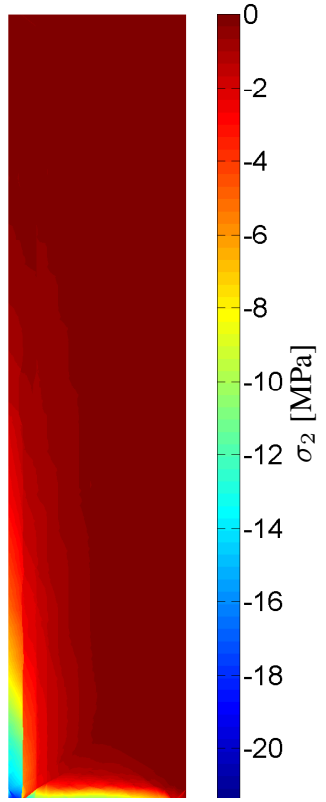

(e)

Figure 9. a) Collapse mode of the wall with the door openings. b) - e) Smallest principal stresses of the four walls of the stairwell example with $\nu=1$ using the fine mesh.

below, which is to be expected. For the first example, the deep beam, the analytical solution is well-known. It is observed from Tab. 1 that the error is approximately inversely proportional to the number of element, i.e. increasing the number of elements by a factor of four decrease the error by a factor of four.

For the cantilever I-beam and stairwell examples, the computed limit loads only increase marginally for the fine meshes compared to the coarse mesh. This indicate that coarse meshes provide reasonable approximations to the actual stress field.

The computational time required for the three examples is illustrated in Figure 10. It is observed that the computational time is approximately proportional to the number of elements to the power of 1.1, indicated by $O\left(n^{1.1}\right)$ in the figure. Moreover, the cantilever I-beam example required a lower computational time due to the use of the von Mises criterion, but the slope of the curve seems to be approximately the same as the other two examples.

\section{Conclusion}

A generalised plane stress element subject to in-plane forces has been presented. The element is a lower bound element with a linear stress distribution. The necessary equilibrium equations of

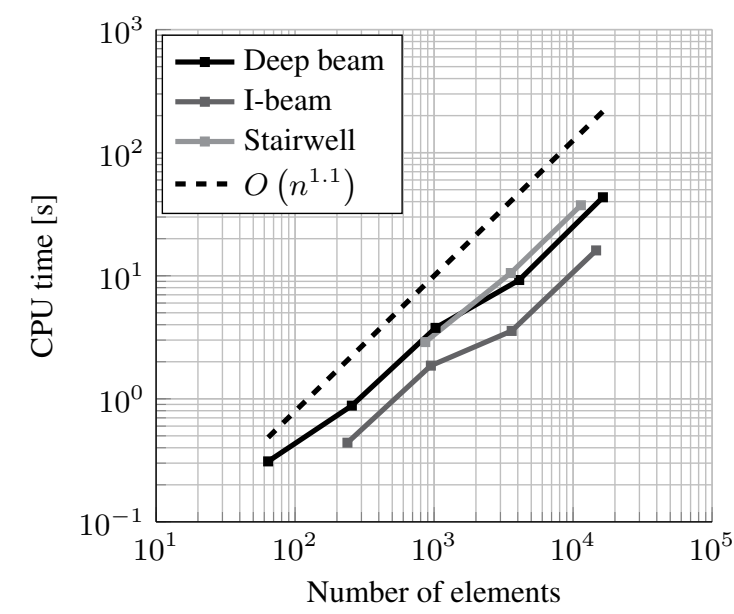

Figure 10. Computational time as a function of the number of elements.

the element are presented together with two different yield criteria, namely the Mohr-Coulomb criterion with a tension cut-off and the von Mises criterion, both for plane stress. Both yield criteria fit the format of second-order cone programming, a class of optimisation problems which can be solved efficiently using interior point methods. 
Herfelt et al.

Three examples are presented to display the use of the element. The first example is a plane deep beam with shear supports subject to a uniformly distributed load. The analytical solution is wellknown and the model approaches the true limit load from below as the mesh density is increased. The next example is a steel cantilever I-beam which uses the von Mises criterion. Again, the model approaches the limit load from below.

The final example is a four-storey stairwell with door openings subject to a shear force which introduces bending and torsion. The limit load is determined using three different meshes using two different effectiveness factors. Stress concentrations are observed near the door openings, however, they are not critical. The collapse mode is illustrated using the solution of the dual problem.

The presented element is capable of modelling complex structures with a satisfactory accuracy. Moreover, it produces a lower bound value and approaches the limit load from below. The computational time is more or less proportional to the problem size, thus, even large problems can be solved in a matter of minutes on a standard laptop.

\section{Acknowledgement}

The authors would like to thank the ALECTIA Foundation and Innovation Fund Denmark for financial support of the research presented in this paper.

\section{REFERENCES}

Anderheggen E and Knöpfel H (1972) Finite element limit analysis using linear programming. International Journal of Solids and Structures 8: 1413-1431.

Andersen ED, Roos C and Terlaky T (2003) On implementing a primal-dual interior-point method for conic quadratic optimization. Mathematical Programming 95(2): 249-277.

Bisbos C and Pardalos P (2007) Second-order cone and semidefinite representations of material failure criteria. Journal of Optimization Theory and Applications 134(2): 275-301.

Boyd SP and Vandenberghe L (2004) Convex Optimization. Cambridge University Press.

Drucker D, Prager W and Greenberg H (1952) Extended limit design theorems for continuous media. Quarterly of Applied Mathematics 9: 381-389.

Herfelt MA, Poulsen PN, Hoang LC and Jensen JF (2016)

Numerical limit analysis of keyed shear joints in concrete structures. Structural Concrete 17(3): 481-490.
Ingerslev Å (1921) Om en elementær beregningsmetode for krydsarmerede plader. Ingeniфren 30(69): 507-515, translation: On an elementary calculation method for two-way slabs. Johansen KW (1962) Yield-line theory. Cement and Concrete Association.

Karmarkar N (1984) A new polynomial-time algorithm for linear programming. Combinatorica 4: 373-395.

Krabbenhøft K, Lyamin A and Sloan S (2007) Formulation and solution of some plasticity problems as conic programs. International Journal of Solids and Structures 44(5): 1533-1549.

Larsen KP (2010) Numerical Limit Analysis of Reinforced Concrete Structures. PhD thesis, Technical University of Denmark.

Lobo MS, Vandenberghe L, Boyd S and Lebret H (1998) Applications of second-order cone programming. Linear algebra and its applications 284(1): 193-228.

Lyamin AV, Sloan SW, Krabbenhoft K and Hjiaj M (2005) Lower bound limit analysis with adaptive remeshing. International Journal for Numerical Methods in Engineering 63(14): 1961-1974.

Makrodimopoulos A and Martin C (2006) Lower bound limit analysis of cohesive-frictional materials using second-order cone programming. International Journal for Numerical Methods in Engineering 66(4): 604-634.

Makrodimopoulos A and Martin C (2007) Upper bound limit analysis using simplex strain elements and second-order cone programming. International Journal for Numerical and Analytical Methods in Geomechanics .

MOSEK ApS (2015) The MOSEK optimization toolbox for MATLAB manual. Version 7.1 (Revision 33). https: / / www. mosek.com/.

Muttoni A, Schwartz J and Thürlimann B (1997) Design of Concrete Structures With Stress Fields. Springer.

Nesterov Y, Todd MJ and Ye Y (1999) Infeasible-start primal-dual methods and infeasibility detectors for nonlinear programming problems. Mathematical Programming 84(2): 227-267.

Nesterov YE and Todd MJ (1997) Self-scaled barriers and interior-point methods for convex programming. Mathematics of Operations research 22(1): 1-42.

Nielsen MP (1971) On the strength of reinforced concrete discs. Civil engineering and building construction series no.70, Acta 
polytechnica scandinavica.

Nielsen MP and Hoang LC (2010) Limit Analysis and Concrete

Plasticity, Third Edition. Taylor \& Francis.

Poulsen PN and Damkilde L (2000) Limit state analysis of reinforced concrete plates subjected to in-plane forces.

International Journal of Solids and Structures 37: 6011-6029.

Prager W (1952) The general theory of limit design. In Proceedings of the 8th International Congress on theoretical and Applied Mechanics, Istanbul, vol. 19, pp. 65-72.

Ribó R, Pasenau M, Escolano E, Ronda J and González L (1998) Gid reference manual. CIMNE, Barcelona .

Sloan SW (1988) Lower bound limit analysis using finite elements and linear programming. International Journal for Numerical and Analytical Methods in Geomechanics 12: 61-77.

Smith C, Gilbert M, Hawksbee S and Babiker A (2014) Recent advances in the application of discontinuity layout optimization to geotechnical limit analysis problems. Numerical Methods in Geotechnical Engineering 1: 415.

Sturm JF (1997) Primal-dual interior point approach to semidefinite programming. $\mathrm{PhD}$ thesis, Thesis Publishers Amsterdam, The Netherlands.

Terlaky T (2013) Interior point methods of mathematical programming, vol. 5. Springer Science \& Business Media. 